\title{
A UNIFORMLY CONVERGENT SERIES FOR STURM-LIOUVILLE EIGENVALUES*
}

BY

\author{
DAVIS COPE
}

North Dakota State University

\begin{abstract}
For the regular Sturm-Liouville problem with equation $y^{\prime \prime}+(\lambda-q(x)) y=0$ on $0 \leqslant x \leqslant \pi$, there are well-known asymptotic expansions for the eigenvalues and eigenfunctions. We show that these asymptotic expansions can be replaced by convergent series for sufficiently large eigenvalues. Convergence is uniform on the interval $0 \leqslant x \leqslant \pi$ and uniform with respect to the eigenvalues, in the sense that a single majorant bounds all series. The basic idea is to replace the asymptotic results, which use an expansion of powers of $n^{-1}$ or $(n+1 / 2)^{-1}$ for integers $n$, by a series in powers of $\mu^{-1}$, where $\mu^{2}$ is an eigenvalue for the corresponding constant coefficient Sturm-Liouville problem with equation $y^{\prime \prime}+\lambda y=0$.
\end{abstract}

1. Introduction. Consider the regular Sturm-Liouville problem

$$
\begin{aligned}
y^{\prime \prime}+(\lambda-q(x)) y & =0 \quad \text { on } 0 \leqslant x \leqslant \pi, \\
y(0) \cos \alpha+y^{\prime}(0) \sin \alpha & =0, \\
y(\pi) \cos \beta+y^{\prime}(\pi) \sin \beta & =0,
\end{aligned}
$$

$q(x)$ real and piecewise continuous. There are well-known asymptotic expansions for large eigenvalues and the corresponding eigenfunctions. There are two customary ways of obtaining such expansions: using the integral equation for (1) directly - see Titchmarsh (1962) and Levitan and Sargsjan (1975) - or first performing a modified Prüfer substitution - see Hochstadt (1961) notes that the Prüfer substitution provides a rigorous foundation for the WKB method. This substitution also has computational advantages and is used in Fix (1967) to obtain recurrence formulas for higher order terms in the eigenvalue expansions. Both methods lead to similar asymptotic expansions. Levitan and Sargsjan (1975) obtain for sufficiently large $n$ and for $q(x)$ with a bounded derivative:

$\sin \alpha=\sin \beta=0$ :

$$
\begin{aligned}
& \lambda^{1 / 2} \sim n+\frac{c}{\pi n}+O\left(\frac{1}{n^{2}}\right) \\
& y(x) \sim \sin (n x)+O\left(\frac{1}{n}\right) .
\end{aligned}
$$

\footnotetext{
${ }^{*}$ Received November 22, 1983.
} 
$\sin \alpha=0, \sin \beta \neq 0$ :

$$
\begin{gathered}
\lambda^{1 / 2} \sim n+\frac{1}{2}+\frac{c+\cot \beta}{\pi\left(n+\frac{1}{2}\right)}+O\left(\frac{1}{n^{2}}\right), \\
y(x) \sim \sin \left(\left(n+\frac{1}{2}\right) x\right)+O\left(\frac{1}{n}\right) .
\end{gathered}
$$

$\sin \alpha \neq 0$ :

$$
\begin{gathered}
\lambda^{1 / 2} \sim n+\frac{1}{\pi n}(c+\cot \beta-\cot \alpha)+O\left(\frac{1}{n^{2}}\right) \\
y(x) \sim \cos (n x)+\frac{1}{n}\left(-c x-\cot \alpha+\frac{1}{2} \int_{0}^{x} q(t) d t\right) \sin (n x)+\mathcal{O}\left(\frac{1}{n^{2}}\right)
\end{gathered}
$$

where $c=\frac{1}{2} \int_{0}^{\pi} q(t) d t$.

The error terms are bounded uniformly in $x$ on $0 \leqslant x \leqslant \pi$. These authors also note that "the asymptotic formulas for the eigenvalues and eigenfunctions are classical, and are basically due to Liouville" (Levitan and Sargsjan (1975), p. 99).

This paper constructs series expansions for large eigenvalues and corresponding eigenfunctions. These series converge uniformly on $0 \leqslant x \leqslant \pi$ for all sufficiently large eigenvalues. Each term of the series satisfies the boundary conditions of (1) (this is only approximately true for the asymptotic forms in (2)). The convergence is uniform with respect to the size of the eigenvalues in the sense that a single majorant series bounds the expansions for all large eigenvalues; a single majorant also bounds the eigenfunction expansions. The basic idea of the proof is to replace expansions in powers of $n^{-1}$ for integer $n$ with expansions in powers of $\mu^{-1}$, where $\mu^{2}$ is an eigenvalue of the constant coefficient Sturm-Liouville problem (1), that is, with equation $y^{\prime \prime}+\lambda y=0$. These eigenvalues are simple to calculate. The initial terms of these convergent series have the form

$$
\begin{gathered}
\lambda=\mu^{2}+c_{0}+O\left(\frac{1}{\mu}\right), \\
y(x)=\sin \left(\mu x-\phi_{1}\right)+\frac{1}{\mu} \int_{0}^{x}\left(\sin \left(\mu t-\phi_{1}\right)\right)^{2}\left(q(t)-c_{0}\right) d t+O\left(\frac{1}{\mu^{2}}\right),
\end{gathered}
$$

where

$$
c_{0}=\frac{2}{\pi+\frac{1}{2 \mu}\left(\sin \left(2 \phi_{2}\right)-\sin \left(2 \phi_{1}\right)\right)} \int_{0}^{\pi}\left(\sin \left(\mu t-\phi_{1}\right)\right) q(t) d t,
$$

where $\phi_{1}, \phi_{2}$ are defined by $\mu, \alpha, \beta$ (see equation (5)).

Section 2 gives the formal construction of the series for the eigenvalue $\lambda$ and eigenfunction $y(x)$ for (1). Section 3 constructs the majorant series. The convergence results are summarized in the theorem at the end of Sec. 3.

2. The expansion. Let $\mu^{2}$ be an eigenvalue for the Sturm-Liouville problem (1) with $q(x) \equiv 0$ on $0 \leqslant x \leqslant \pi$. We take $\mu>0$ and note that it satisfies the equation

$$
\tan \mu \pi=\frac{\mu \sin (\alpha-\beta)}{\mu^{2} \sin \alpha \sin \beta+\cos \alpha \cos \beta} .
$$


We define $\phi_{1}(\mu)$ and $\phi_{2}(\mu)$ by

$$
\begin{array}{ll}
\sin \phi_{1}=\mu \sin \alpha\left[(\cos \alpha)^{2}+(\mu \sin \alpha)^{2}\right]^{-1 / 2}, & \left|\phi_{1}\right| \leqslant \pi / 2, \\
\sin \phi_{2}=\mu \sin \beta\left[(\cos \beta)^{2}+(\mu \sin \beta)^{2}\right]^{-1 / 2}, & \left|\phi_{2}\right| \leqslant \pi / 2 .
\end{array}
$$

Equation (4) can be written as

$$
\tan \mu \pi=\tan \left(\phi_{1}-\phi_{2}\right),
$$

and therefore, given $\mu$, there is a unique integer $N(\mu)$ such that

$$
\mu \pi=N(\mu) \pi+\phi_{1}(\mu)-\phi_{2}(\mu) .
$$

The eigenfunction $E(x)$ corresponding to $\mu^{2}$ can be written

$$
E(x)=\sin \left(\mu x-\phi_{1}\right)=(-1)^{N(\mu)+1} \sin \left(\mu(\pi-x)+\phi_{2}\right),
$$

which has been normalized so that $|E(x)| \leqslant 1$.

Let $\lambda=\mu^{2}+c$ be an eigenvalue of the full Sturm-Liouville system (1). Then (1) can be written as

$$
y^{\prime \prime}+\mu^{2} y=(q(x)-c) y,
$$

with the corresponding integral equation chosen as

$$
y(x)=E(x)+\frac{1}{\mu} \int_{0}^{x} \sin (\mu(x-t))(q(t)-c) y(t) d t .
$$

Each solution of the integral equation satisfies the differential equation in (1) and the boundary condition at $x=0$. The eigenvalue problem can be restated as finding the constant $c$ so that the solution to (7) satisfies the boundary condition at $x=\pi$.

We introduce the series expansions

$$
Y(x)=\sum_{k=0}^{\infty} u_{k}(x) \mu^{-k}, Y^{\prime}(x)=\sum_{k=0}^{\infty} v_{k}(x) \mu^{-k+1}, c=\sum_{k=0}^{\infty} c_{k} \mu^{-k} .
$$

Substitution of the series for $Y(x)$ and for $c$ into the integral equation (7) and matching powers of $\mu^{-1}$ yields the recursion relations for $u_{k}(x)$ :

$$
\begin{aligned}
& u_{0}(x)=E(x)=\sin \left(\mu x-\phi_{1}\right) \\
& u_{k}(x)=\int_{0}^{x} \sin (\mu(x-t))\left[\left(q(t)-c_{0}\right) u_{k-1}(t)-c_{1} u_{k-2}(t)-\cdots-c_{k-1} u_{0}(t)\right] d t
\end{aligned}
$$

for $k \geqslant 1$.

The $v_{k}(x)$ are defined by termwise differentiation of the $u_{k}(x)$ :

$$
\begin{aligned}
& \mu v_{0}(x)=\mu \cos \left(\mu x-\phi_{1}\right), \\
& \mu v_{k}(x)=\mu \int_{0}^{x} \cos (\mu(x-t))\left[\left(q(t)-c_{0}\right) u_{k-1}(t)-\cdots-c_{k-1} u_{0}(t)\right] d t
\end{aligned}
$$

for $k \geqslant 1$.

Observe that $u_{0}(x)$ satisfies the boundary conditions at both $x=0$ and $x=\pi$, and that each $u_{k}(x)$ satisfies the boundary conditions at $x=0$ (trivially, since $u_{k}(0)=u_{k}^{\prime}(0)=0$ 
for $k \geqslant 1)$. We choose the values $c_{k}$ so that each $u_{k}(x)$ satisfies the boundary condition at $x=\pi$, that is, we choose $c_{k-1}$ such that, for $k \geqslant 1$,

$$
\begin{aligned}
0=\int_{0}^{\pi}[ & \sin (\mu(\pi-t)) \cos \beta+\mu \cos (\mu(\pi-t)) \sin \beta] \\
& \times\left[\left(q(t)-c_{0}\right) u_{k-1}(t)-\cdots-c_{k-1} u_{0}(t)\right] d t,
\end{aligned}
$$

or, using (6), the equivalent

$$
0=\int_{0}^{\pi} \sin \left(\mu t-\phi_{1}\right)\left[\left(q(t)-c_{0}\right) u_{k-1}(t)-\cdots-c_{k-1} u_{0}(t)\right] d t .
$$

The equations for $c_{k}$ become

$$
\begin{gathered}
c_{0}=A(\mu) \int_{0}^{\pi}\left(\sin \left(\mu t-\phi_{1}\right)\right)^{2} q(t) d t \\
c_{k}=A(\mu) \int_{0}^{\pi} \sin \left(\mu t-\phi_{1}\right)\left[\left(q(t)-c_{0}\right) u_{k}(t)-\cdots c_{k-1} u_{1}(t)\right] d t \text { for } k \geqslant 1,
\end{gathered}
$$

where

$$
A(\mu)=\frac{2}{\pi+\left(\sin \left(2 \phi_{2}\right)-\sin \left(2 \phi_{1}\right)\right) / 2 \mu} .
$$

For use in the next section we note the bounds

$$
\begin{aligned}
& \text { If } \sin (\beta-\alpha)=0, \text { then }|A(\mu)|=2 / \pi \text {. } \\
& \text { If } \sin (\beta-\alpha) \neq 0 \text {, then for each } \delta>0 \text {, } \\
& \text { if } \mu>(2+\delta) / \delta \pi \text {, then }|A(\mu)|<(2+\delta) / \pi \text {. }
\end{aligned}
$$

The series for $Y(x), Y^{\prime}(x)$, and $c$ in (8) are now defined. In the next section we prove these series absolutely and uniformly convergent on $0 \leqslant x \leqslant \pi$ for all sufficiently large $\mu$. These convergence results imply that $Y^{\prime}(x)$ can be integrated termwise to yield $Y(x)$ as its antiderivative and that termwise integration as performed in (9) is permissible, so that the series for $Y(x)$ and $c$ can be combined to yield a solution of the integral equation (8). Also, $Y(x)$ and $Y^{\prime}(x)$ satisfy the boundary conditions at both $x=0$ and $x=\pi$ because their individual terms do so. Consequently, $\mu^{2}+c$ and $Y(x)$ represent an eigenvalue and eigenfunction for the Sturm-Liouville problem (1).

3. Proof of convergence. Pick an arbitrary $\delta>0$. If $\sin (\beta-\alpha) \neq 0$, assume $\mu>(2+$ $\delta) / \delta \pi$; if $\sin (\beta-\alpha)=0$, simply retain the assumption that $\mu>0$ and take $\delta=0$ in the following arguments. These assumptions permit us to use the bounds of equation (12), and the recursion relations of (9), (10) and (11) then yield the bounds

$$
\begin{gathered}
\left|u_{0}(x)\right| \leqslant 1, \\
\left|u_{k}(x)\right|,\left|v_{k}(x)\right| \leqslant \int_{0}^{x}\left|q(t)-c_{0}\right|\left|u_{k-1}(t)\right|+\cdots+\left|c_{k-1}\right|\left|u_{0}(t)\right| d t \quad \text { for } k \geqslant 1, \\
\left|c_{k}\right| \leqslant \frac{2+\delta}{\pi} \int_{0}^{\pi}\left|q(t)-c_{0}\right|\left|u_{k}(t)\right|+\cdots+\left|c_{k-1}\right|\left|u_{1}(t)\right| d t
\end{gathered}
$$


Introduce the series

$$
\begin{aligned}
P(x, z) & =\sum_{k=0}^{\infty} P_{k}(x) z^{k}, \quad Q(z)=\sum_{k=0}^{\infty} Q_{k} z^{k} \\
P_{0}(x) & =1, \quad Q_{0}=\max _{0 \leqslant x \leqslant \pi}\left|q(x)-c_{0}\right| \\
P_{k}(x) & =\int_{0}^{x} Q_{0} P_{k-1}(t)+\cdots+Q_{k-1} P_{0}(t) d t \quad \text { for } k \geqslant 1, \\
Q_{k} & =\frac{2+\delta}{\pi} \int_{0}^{\pi} Q_{0} P_{k}(t)+\cdots+Q_{k-1} P_{1}(t) d t \quad \text { for } k \geqslant 1 .
\end{aligned}
$$

Comparison of (13) and (14) shows that $\left|u_{k}(x)\right|,\left|v_{k}(x)\right| \leqslant P_{k}(x)$ and $\left|c_{k}\right| \leqslant Q_{k}$ for $k \geqslant 1$. Also, each coefficient $P_{k}(x)$ is positive and increasing on $0 \leqslant x \leqslant \pi$. We now show that $P(\pi, z)$ and $Q(z)$ have the same radius of convergence and then calculate the radius exactly. Majorant series for $Y(x), Y^{\prime}(x), c$ in (8) immediately follow. (Incidentally, if we take each coefficient $P_{k}(x)$ constant and set up relations for $P(z)$ and $Q(z)$, then $P(z)$ and $Q(z)$ can be found explicitly and the radius of convergence is easily obtained. However, the radius found by this method is roughly half the radius obtained by the following argument.)

The recursion relations (14) can be written as

$$
\begin{gathered}
P(x, z) 1=z Q(z) \int_{0}^{x} P(t, z) d t, \\
Q(z)-Q_{0}=\frac{2+\delta}{\pi} Q(z) \int_{0}^{\pi}(P(t, z)-1) d t .
\end{gathered}
$$

Solving the first of these gives

$$
P(x, z)=\exp (z Q(z) x) .
$$

Then $P(\pi, z)=\exp (\pi z Q(z))$ has the same radius of convergence as $Q(z)$. Substitution of (16) into the second equation of (15) gives

$$
\zeta=(3+\delta) \omega-(2+\delta)\left(e^{\omega}-1\right)
$$

where $\zeta=\pi Q_{0} z, \omega=\pi z Q(z), \delta \geqslant 0$.

We wish to find the radius of convergence of the solution $\omega=\zeta+0\left(\zeta^{2}\right)$ about $\zeta=0$. Equation (17) cannot be solved explicitly for $\omega(\zeta)$ but the radius can still be found as follows:

First, observe that for $\omega$ real, $\zeta(\omega)$ is real, strictly increasing for $\omega<\omega_{0}$, strictly decreasing on $\omega_{0}<\omega$, with maximum $\zeta_{0}$ at $\omega_{0}$ :

$$
\begin{gathered}
\omega_{0}=\ln \left(\frac{3+\delta}{2+\delta}\right)>0, \\
\zeta_{0}=(3+\delta) \ln \left(\frac{3+\delta}{2+\delta}\right)-1>0 .
\end{gathered}
$$

The maximum value for the radius of convergence of $\omega(\zeta)$ is therefore $|\zeta|<\zeta_{0}$, since $\omega(\zeta)$ becomes double-valued at this point. We now show that $\zeta_{0}$ is the actual value for the radius. 
First, it will be shown that $\zeta(\omega)$ is one-to-one on the closed set $S: S=\left\{\omega: \operatorname{Re}(\omega) \leqslant \omega_{0}\right\}$. Recall the result from the theory of functions of a complex variable:

THEOREM (Copson (1935), pp. 134-135). Let $w=f(z)$ be analytic inside and on the simple closed curve $C$ and assume $f(z)$ is one-to-one on $C$. Then $f(C)$ is a simple closed curve, and $w=f(z)$ is a one-to-one map from $C$ and its interior to $f(C)$ and its interior.

The proof is a straightforward application of the argument principle.

We apply this theorem to the map $\zeta=\zeta(\omega)$ and the curves $C_{n}$, defined for positive integers $n$ as the boundary of the sets $S_{n}$,

$$
\begin{aligned}
S_{n}:-n \ln 2 \leqslant \operatorname{Re}(\omega) \leqslant \omega_{0}, \\
-2 n \pi \leqslant \operatorname{Im}(\omega) \leqslant+2 n \pi .
\end{aligned}
$$

If $\zeta(\omega)$ is one-to-one on each curve $C_{n}$ for sufficiently large $n$, then $\zeta(\omega)$ is one-to-one on $S_{n}$ by the above theorem. Letting $n \rightarrow+\infty, \zeta(\omega)$ is then one-to-one on $S$.

To show that $\zeta(\omega)$ is one-to-one on $C_{n}$, write out the real components of (17) for each edge. Using $\zeta=x+i y, \omega=u+i v$ :

On the lower edge of $C_{n}$.

$$
\begin{gathered}
x=(3+\delta) u-(2+\delta)\left(e^{u}-1\right), \\
y=-2 n \pi(3+\delta), \\
-n \ln 2 \leqslant u \leqslant \omega_{0} .
\end{gathered}
$$

On the right edge of $C_{n}$

$$
\begin{gathered}
x=(3+\delta) \ln \left(\frac{3+\delta}{2+\delta}\right)+(2+\delta)-(3+\delta) \cos v, \\
y=(+\delta)(v-\sin v) \\
\quad-2 n \pi \leqslant v \leqslant+2 n \pi .
\end{gathered}
$$

On the upper edge of $C_{n}$

$$
\begin{gathered}
x=(3+\delta) u-(2+\delta)\left(e^{u}-1\right), \\
y=+2 n \pi(3+\delta), \\
-n \ln 2 \leqslant u \leqslant \omega_{0} .
\end{gathered}
$$

On the left edge of $C_{n}$

$$
\begin{gathered}
x=-(3+\delta) n \ln 2+(2+\delta)\left(1-2^{-n} \cos v\right), \\
y=(3+\delta) v-(2+\delta) 2^{-n} \sin v, \\
-2 n \pi \leqslant v \leqslant+2 n \pi .
\end{gathered}
$$

Proceeding counterclockwise around $C_{n}$, the image can be described as follows: Using (19), the image in the $\zeta$-plane of the lower edge of $C_{n}$ is a straight line segment with $y$ constant and $x$ strictly increasing. Using (20), the image of the right edge of $C_{n}$ is a curve with $y$ strictly increasing and $x$ oscillating between a minimum of $\zeta_{0}>0$ and a maximum of $\zeta_{0}+2(3+\delta)$. Using (21), the image of the upper edge of $c_{n}$ (continuing counterclockwise around $C_{n}$ ) is a straight line segment with $y$ constant and $x$ strictly decreasing. Using (22), the image of the left edge of $C_{n}$ (continuing counterclockwise) is a curve with $y$ strictly decreasing and $x$ oscillating between $x_{1}=-(3+\delta) n \ln 2+(2+\delta)\left(1-2^{-n}\right)$ and 
$x_{1}+2^{-n+1}(\mathrm{~d}+\delta)$. For sufficiently large $n$ it is clear that the images of different edges (in particular, the left and right edges) cannot overlap. It follows that $\zeta(\omega)$ is one-to-one on $C_{n}$ for all sufficiently large $n$, and as noted above $\zeta(\omega)$ is therefore one-to-one on $S$.

Since $\zeta(\omega)$ is one-to-one on $S$, which contains $\omega=0$ in its interior, then $\omega(\zeta)$ exists as an analytic function on the interior of the set $\zeta(S)$, which contains $\zeta=0$ in its interior. The boundary of $\zeta(S)$ is the image of the curve $\operatorname{Re}(\omega)=\omega_{0}$, and the equations of this boundary are given by (20) with $-\infty<v+\infty$. This curve is symmetric about the $x$-axis, and the $x$-values oscillate between $x=\zeta_{0}$ and $x=\zeta_{0}+2(3+\delta)$. It is easily seen that the closest point on this boundary curve to the origin $\zeta=0$ is the point $\zeta=\zeta_{0}$. Consequently, $\zeta(S)$ contains the disc $|\zeta|<\zeta_{0}, \omega(\zeta)$ is analytic on this disc, and the radius of convergence for $\omega(\zeta)$ about $\zeta=0$ is therefore at least $\zeta_{0}$. In connection with (18), it was noted that the radius is at most $\zeta_{0}$. Consequently, the radius of convergence of the solution $\omega(\zeta)=\zeta+$ $O\left(\zeta^{2}\right)$ of $(17)$ is exactly $\zeta_{0}(\delta)$, as defined in (18).

We now summarize the convergence results. The series

$$
\sum P_{k}(\pi) \mu^{-k}, \quad \sum P_{k}(\pi) \mu^{-k+1}, \quad \sum Q_{k} \mu^{-k},
$$

with coefficients defined in (14), are majorants for the series $Y(x), Y^{\prime}(x)$, and $c$ of (8). These majorant series all have the same radius of convergence which can be summarized as:

$$
\begin{aligned}
& \text { If } \sin (\beta-\alpha)=0 \text {, then convergence for all } \\
& \mu>\pi Q_{0}\left[3 \ln \left(\frac{3}{2}\right)-1\right]^{-1} \text {, that is, all } \mu>14.52 Q_{0} .
\end{aligned}
$$

If $\sin (\beta-\alpha) \neq 0$, then convergence for all $\mu$ such that

$$
\mu>\frac{2+\delta}{\delta \pi} \text { and } \mu>\pi Q_{0}\left[(3+\delta) \ln \left(\frac{3+\delta}{2+\delta}\right)-1\right]^{-1} .
$$

In the condition for $\sin (\beta-\alpha) \neq 0$, one function of $\delta$ is strictly decreasing on $\delta>0$ and the other is strictly increasing. The point of intersection of these two functions then corresponds to the largest interval of convergence for $\mu$. Altogether we have:

THEOREM. Let $\mu^{2}$ be an eigenvalue for the Sturm-Liouville problem (1) with $q(x) \equiv 0$, and assume

(a) if $\sin (\beta-\alpha)=0$, then $\mu>14.52 Q_{0}$,

(b) if $\sin (\beta-\alpha) \neq 0$, then $\mu>Q_{1}$, where $Q_{1}$ is the unique value such that

$$
Q_{1}=\frac{2+\delta}{\delta \pi}=\pi Q_{0}\left[(3+\delta) \ln \left(\frac{3+\delta}{2+\delta}\right)-1\right]^{-1}
$$

for some $\delta>0$, and

$$
Q_{0}=\max _{0 \leqslant x \leqslant \pi}\left|q(x)-c_{0}\right|
$$

with $c_{0}$ defined in (11).

Then the series $Y(x), Y^{\prime}(x)$, and $c$ defined in (8)-(11) converge absolutely and uniformly on $0 \leqslant x \leqslant \pi$, and $\lambda=\mu^{2}+c$ is an eigenvalue for the full Sturm-Liouville problem (1) with eigenfunction $y(x)=Y(x)$. 


\section{REFERENCES}

[1] E. T. Copson, Theory of functions of a complex variable, Oxford University Press, London, 1935

[2] G. Fix, Asymptotic eigenvalues of Sturm-Liouville systems, J. Math. Anal. Appl. 19 519-525 (1967)

[3] H. Hochstadt, Asymptotic estimates for the Sturm-Liouville spectrum, Comm. Pure Appl. Math. 14 749-764 (1961)

[4] B. M. Levitan and I. S. Sargsjan, Introduction to spectral theory: selfadjoint ordinary differential operators, Translations of Mathematical Monographs, vol. 39, American Mathematical Society, Providence, R.I., 1975 (Original Russian edition 1970)

[5] E. C. Titchmarch, Eigenfunction expansions associated with second-order differential equations, Part I, Clarendon Press, Oxford, 1962 